\title{
A BIOLOGIC RISK MODEL FOR STAGE I LUNG CANCER: IMMUNOHISTOCHEMICAL ANALYSIS OF 408 PATIENTS WITH THE USE OF TEN MOLECULAR MARKERS
}

\author{
Thomas A. D'Amico, MD \\ Marga Massey, MD \\ James E. Herndon II, PhD \\ Mary-Beth Moore \\ David H. Harpole, Jr, MD
}

Objective: The standard treatment of patients with stage I non-small cell lung cancer is resection of the primary tumor; however, the recurrence rate is $28 \%$ to $45 \%$. This study evaluates a panel of molecular markers in a large population of patients with stage $I$ non-small cell lung cancer to determine the prognostic value of each marker and to create a biologic risk model. Methods: Pathologic specimens were collected from 408 consecutive patients after complete resection for stage I non-small cell lung cancer at a single institution, with follow-up of at least 5 years. A panel of 10 molecular markers was chosen for immunohistochemical analysis of the primary tumor on the basis of differing oncogenic mechanisms. Local tumor expansion requires growth regulating proteins (epidermal growth factor receptor, the protooncogene $e r b$-b2); apoptosis proteins (p53, bcl-2); and cell cycle regulating proteins (retinoblastoma recessive oncogene, KI-67). Local tumor invasion requires angiogenesis (factor viii). The development of distant metastases involves the expression of adhesion proteins (CD-44, sialyl-Tn, blood group A). Cox proportional hazards regression analysis was used to construct an independent risk model for cancer recurrence and death. Results: Multivariable analysis demonstrated significantly elevated risk for the following molecular markers: p53 (hazard ratio, 1.68; $P=.004)$; factor viii (hazard ratio, 1.47; $P=.033$ ); $e r b$-b2 (hazard ratio, $1.43 ; P=.044)$; CD44 (hazard ratio, $1.40 ; P=.050$ ); and retinoblastoma recessive oncogene (hazard ratio, $0.747 ; P=.084)$. Conclusions: Five molecular markers were associated with the risk of recurrence and death, representing independent metastatic pathways: apoptosis (p53), angiogenesis (factor viii), growth regulation ( $e r b-\mathrm{b} 2)$, adhesion (CD-44), and cell cycle regulation (retinoblastoma recessive oncogene). This study demonstrates the validity of this molecular biologic risk model in patients with stage I nonsmall cell lung cancer. (J Thorac Cardiovasc Surg 1999;117:736-43)
$\mathrm{N}$ on-small cell lung cancer (NSCLC) is the most common cause of death by malignancy in both men and women in the United States. ${ }^{1}$ The current staging

From the Thoracic Oncology Program, Duke Comprehensive Cancer Center, Duke University Medical Center, Durham, NC.

Supported by National Cancer Institute grant R29 CA73980.

Read at the Twenty-fourth Annual Meeting of The Western Thoracic Surgical Association, Whistler, British Columbia, June 24-27, 1998.

Received for publication July 15, 1998; revisions requested Sept 8, 1998; revisions received Nov 13, 1998; accepted for publication Dec 10, 1998.

Address for reprints: Thomas A. D'Amico, MD, Duke University Medical Center, Box 3496, Durham, NC 27707.

Copyright (C) 1999 by Mosby, Inc.

$0022-5223 / 99 \$ 8.00+0 \quad \mathbf{1 2 / 6 / 9 6 5 3 5}$ system for NSCLC considers the size and location of the primary tumor $(\mathrm{T})$, the involvement of regional lymph nodes $(\mathrm{N})$, and the presence of distant metastases (M). ${ }^{2}$ The standard treatment of patients with stage I NSCLC (T1-2 N0) is resection of the primary tumor (no adjuvant therapy). However, even after complete resection, 5-year survival is only $55 \%$ to $72 \%$ in this group of patients, predominately because of the development of distant metastases. ${ }^{2-7}$

An operation alone is considered the standard therapy for patients with stage I NSCLC. To select a subgroup of patients with stage I disease who might benefit from adjuvant therapy, investigators have attempted to identify factors that predict poor prognosis, including analysis of performance status, the subtype and size of the primary tumor, the degree of tumor differentiation, the 
mitotic rate, and the evidence of lymphatic or vascular invasion..$^{5,7-13}$ These factors indirectly measure tumor aggressiveness and have not identified a group of stage I patients who benefit from adjuvant therapy.

Recent interest has focused on the identification of biologic markers that predict early recurrence and death in patients with lung cancer. Advances in molecular biology, specifically the study of oncogenes and other molecular markers, have been applied in the development of a prognostic index of recurrence in patients with stage I NSCLC. ${ }^{13-26}$ These serum and tissue markers may be classified by the mechanism of action that results in metastases: growth regulation, cell cycle regulation, apoptosis, angiogenesis, and cellular adhesion. Local tumor expansion requires growth-regulating proteins (epidermal growth factor receptor [EGFr], the protooncogene $e r b-\mathrm{b} 2)$; apoptosis proteins (p53, bcl-2); and cell cycle-regulating proteins (retinoblastoma recessive oncogene [rb], KI-67). Local tumor invasion requires angiogenesis (factor viii). The development of distant metastases involves the expression of adhesion proteins (CD-44, sialyl Tn, blood group A).

Stratification of patients without lymph node involvement, according to a prognostic risk model, might select a group of high-risk patients who would benefit from adjuvant therapy. This study evaluates a panel of molecular biologic markers in patients with stage I NSCLC after complete resection to determine the prognostic value of each marker and to create a biologic risk model. The molecular markers assessed in this study encompass various biologic mechanisms of metastases.

\section{Methods}

From January 1, 1980, until December 31, 1992, 6510 consecutive patients with documented NSCLC were evaluated at the Comprehensive Cancer Center at Duke University Medical Center. Inclusion criteria for entry into the study population included pathologic stage I NSCLC (T1 N0 M0 or T2 N0 M0) by the International System of Staging for Lung Cancer, ${ }^{2}$ complete resection, no adjuvant therapy, minimum 60-day survival, and availability of pathologic material. During this period, 408 patients met these criteria; all patients in this study have been followed for at least 60 months ( 5 years).

Each case was reviewed by a pathologist to ascertain tumor size and cell type and to exclude the involvement of hilar and mediastinal lymph nodes. Only squamous cell, large cell, and adenocarcinoma variants are included in this analysis. The greatest dimension of the tumor was measured in centimeters; the primary lesion was carefully examined for the presence of visceral pleural involvement, lymphatic vessel invasion, and vascular invasion.

Immunohistochemical analyses were performed on 2 paraffin blocks of resected lung tissue for each patient in the study, obtained after approval of the Human Subjects Review Com- mittee protocol. The immunohistochemical analyses have been previously described. ${ }^{13-15}$ In brief, after paraffin microtome sectioning (4 to $6 \mathrm{~mm}$ ), slide labeling, and deparaffinization with xylene and ethanol, antigen retrieval was completed after microwaving and phosphate-buffered saline solution washing. The sections were incubated with primary monoclonal antibody to EGFr, erb-b2, p53, bcl-2, rb, KI-67, factor viii, CD-44 blood group antigen A (BioGenex Laboratories, San Ramon, Calif), and Sialyl-Tn (Dako, Carpinteria, Calif). Incubation with the secondary antibody (horseradish peroxidase) was subsequently performed, followed by development with diaminobenzidine and counterstain with methyl green or hematoxylin.

Immunohistochemical data were recorded without the knowledge of patient outcome. Analysis of p53, rb, bcl-2, EGFr, erb-b2, sialyl-Tn, CD-44, and blood group antigen A were analyzed by 2 independent observers. Known positive tumors and normal lung tissue were used as positive and negative controls, respectively. Blocks were graded positive when widespread staining was present, with an intensity of at least $2(50 \%$ or more positive) on a scale of 0 to 3 . Both observers had to rate a 2 or better for each block to be considered positive. The presence of nuclear staining of p53 was an indication of mutation for the p53 gene. ${ }^{15}$ The KI-67 proliferation index was determined by computerized static image cytometry of 10 consecutive high-powered fields, compared with a background established with control antibody. Analysis of angiogenesis factor viii included the center, the periphery, and the hottest area, with the use of computerized static image cytometry of 10 consecutive high-powered fields.

Statistical analysis. All marker analyses were blinded to patient outcome. The log-rank test and Cox proportional hazards model were used to examine the relationship between cancer-specific survival and various potential prognostic factors individually, comprising immunostaining for rb, EGFr, erb-b2, bcl2, p53, CD-44, blood-group A antigen, sialyl-Tn, and the KI-67 proliferation index and factor viii microvessel count. The joint effect of the variables with $P<.2$ for a univariate association with cancer-death was also examined with the Cox model; the .10 level of significance was used for entering or removing a covariable for this model. Cancer-specific survival was defined as the time between the operation and the last follow-up or cancer death. If a patient died without cancer recurrence, the patient's survival time was censored at the time of death. ${ }^{13-15}$

\section{Results}

The mean age of the patients was $62.9 \pm 8.9$ years. There were 269 men (66\%) and 139 women (34\%; Table I). In this study of 408 patients with pathologically confirmed stage I NSCLC treated by resection alone, there were 149 deaths from cancer; the actual 5year survival was $64 \%$.

There was no significant difference in survival by 
Table I. Expression of molecular biologic markers

\begin{tabular}{llc}
\hline Oncogenic group & \multicolumn{1}{c}{ Marker } & No. positive (\%) \\
\hline Growth factor & EGFr & $212(52)$ \\
& erb-b2 & $104(25)$ \\
Cell cycle factor & rb (normal) & $228(56)$ \\
& KI-67 (elevated) & $205(50)$ \\
Apoptosis factor & p53 & $176(43)$ \\
& bcl-2 & $95(23)$ \\
Angiogenesis factor & Factor viii & $246(60)$ \\
Metastatic adhesion factor & CD-44 & $132(32)$ \\
& Sialyl Tn & $170(42)$ \\
& Blood group & $63 / 101(62)^{*}$ \\
& antigen A & \\
\hline
\end{tabular}

*Thirty-eight percent of the 101 patients with blood type A.

Table II. Univariate survival analysis for molecular biologic markers

\begin{tabular}{llccc}
\hline & & \multicolumn{2}{c}{ 5-Year survival (\%) } & \\
\cline { 3 - 4 } Oncogenic group & Marker & Positive & Negative & P value \\
\hline Growth factor & EGFr & 63 & 61 & .6 \\
& erb-b2 & 47 & 67 & $.002^{*}$ \\
Cell cycle factor & rb & 64 & 60 & $.2^{*}$ \\
& KI-67 & 58 & 66 & $.06^{*}$ \\
Apoptosis factor & p53 & 52 & 70 & $.001^{*}$ \\
& bcl-2 & 64 & 61 & .5 \\
Angiogenesis factor & Factor viii & 56 & 70 & $.008^{*}$ \\
Metastatic adhesion & CD-44 & 54 & 66 & $.02^{*}$ \\
factor & Sialyl Tn & 60 & 63 & .6 \\
& Blood group & 75 & 67 & .4 \\
& antigen A & & & \\
\hline
\end{tabular}

*Significant at $P \leq .2$.

histologic evidence or by the method of resection. Expression of the molecular biologic markers ranged from $23 \%$ to $60 \%$ (Table I). Univariate analysis of the relationship between survival and the panel of molecular biologic markers was statistically significant for p53, erb-b2, rb, KI-67, factor viii, and CD-44 (Table II). Multivariable analysis (Table III) demonstrated significantly elevated risk for the following molecular markers: p53 (Fig 1), factor viii (Fig 2), erb-b2 (Fig 3), CD-44 (Fig 4); and rb (Fig 5).

With multivariable analysis of the entire panel of molecular markers, a prognostic risk model for survival demonstrates significantly decreased survival according to the total number of factors involved (Fig 6). Furthermore, analysis of only the factors demonstrated to be independent multivariable risk factors similarly demonstrates that survival is related to the number of factors involved (Fig 7). There was no significant difference in the prognostic information provided by the
Table III. Multivariable survival analysis for molecular biologic markers

\begin{tabular}{llcl}
\hline Group & Marker & Hazard ratio & P value \\
\hline Growth factor & erb-b2 & 1.429 & .044 \\
Cell cycle factor & rb & 0.747 & .0838 \\
Apoptosis factor & p53 & 1.63 & .037 \\
Angiogenesis factor & Factor viii & 1.47 & .033 \\
Metastatic adhesion factor & CD-44 & 1.399 & .05 \\
\hline
\end{tabular}

molecular marker analysis among patients with $\mathrm{T} 1$ tumors, as compared with the entire population (Fig 8).

\section{Discussion}

The goals of this study were to evaluate a panel of molecular biologic markers in a large, single-institution population of patients with stage I NSCLC after resection and to develop a prognostic model based on the involvement of these molecular variables, independent of other variables. The development of a risk model for patients with stage I disease, in whom the failure rate for an operation alone is $28 \%$ to $45 \%,,^{2-7}$ might eventually be used to select a subgroup of patients who would benefit from adjuvant therapy.

Conventional histopathologic variables have been investigated to determine the prognosis and to assign the therapy, such as the size and subtype of the primary tumor and the degree of differentiation, mitotic rate, and evidence of vascular invasion. Among patients with stage I NSCLC (T1 N0 or T2 N0), there is a clear 5 -year survival advantage for patients with $\mathrm{T} 1(67 \%)$ as compared with T2 $(57 \%)$ disease. ${ }^{2}$ This relationship is consistent for both size $(\leq 3 \mathrm{~cm})$ and location (no pleural involvement). ${ }^{17}$ This difference in survival stimulated the latest modification of the international staging system, which categorizes T1 N0 M0 as stage IA and T2 N0 M0 as stage IB. Nevertheless, this magnitude of this survival difference is overshadowed by the survival differences between stages I, II, and III. The administration of adjuvant therapy has not yet been shown to benefit patients with stage I disease, although this issue is the focus of ongoing multi-institutional cooperative group trials.

The relationship of the degree of differentiation of the primary tumor and survival has been analyzed in patients with stage I NSCLC. ${ }^{13}$ Poorly differentiated or undifferentiated tumors are defined as negative prognostic factors by univariate analysis, but not multivariable analysis. Mitotic rate and pulmonary vascular invasion have been demonstrated to be negative prognostic risk factors; however, this variable, like other 


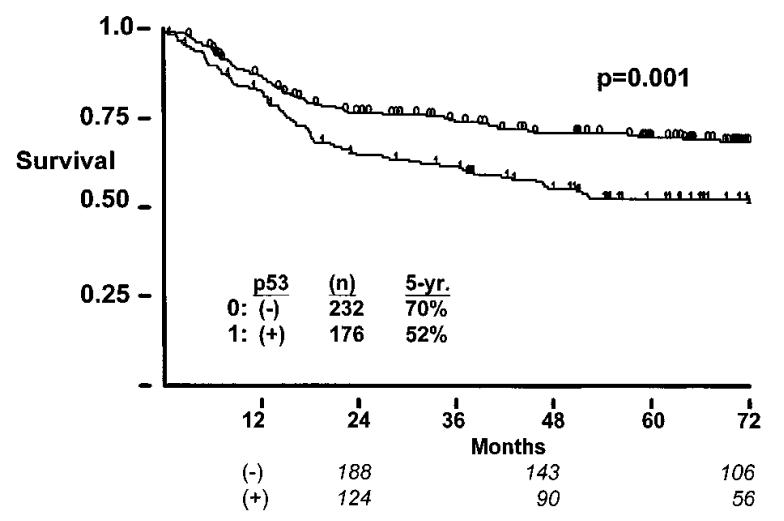

Fig 1. Kaplan-Meier analysis of cancer-specific survival for p53.

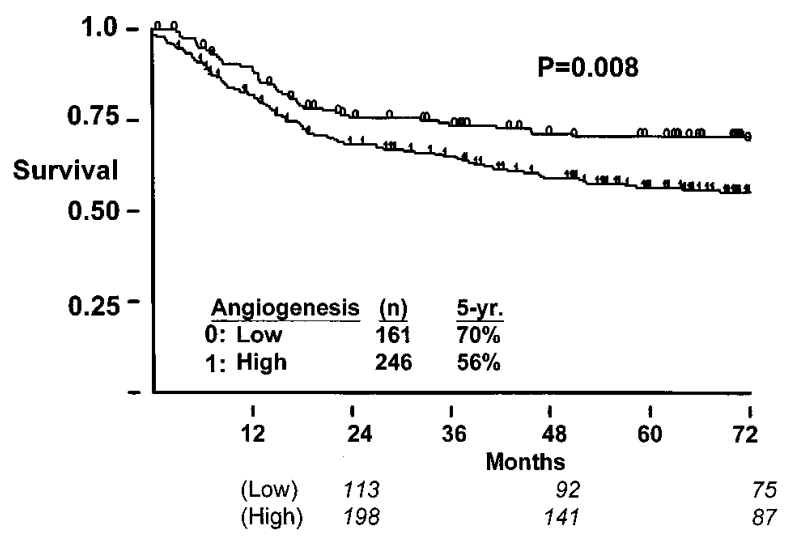

Fig 2. Kaplan-Meier analysis of cancer-specific survival for factor viii.

histopathologic variables, is found to be present in a minority of patients with stage I disease. ${ }^{9,13}$ The use of histopathologic variables (tumor size and subtype, mitotic rate, degree of differentiation, and vascular invasion) to construct a risk model is limited by the low prevalence and the discontinuous nature of the variables.

Molecular biologic markers have recently been studied in lung cancer to determine prognostic importance. Matsuyama and colleagues ${ }^{16}$ demonstrated the prognostic significance of angiogenesis using the immunohistochemical identification of CD34, in which angiogenesis was an independent predictor of death and the presence of hematogenous metastases. This study was not specific to stage I disease, and only 1 molecular marker was studied. Duarte and associates ${ }^{18}$ compared factor viii to CD31 in determining the degree of angiogenesis in patients with stage I disease. This study

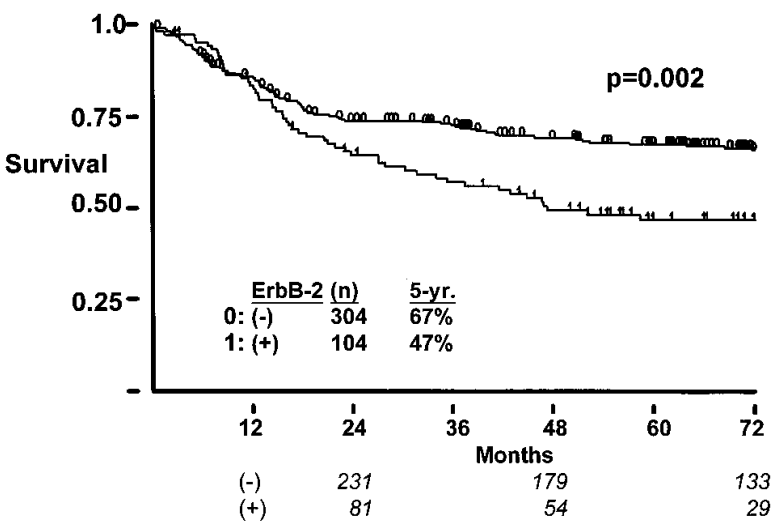

Fig 3. Kaplan-Meier analysis of cancer-specific survival for erb-b2.

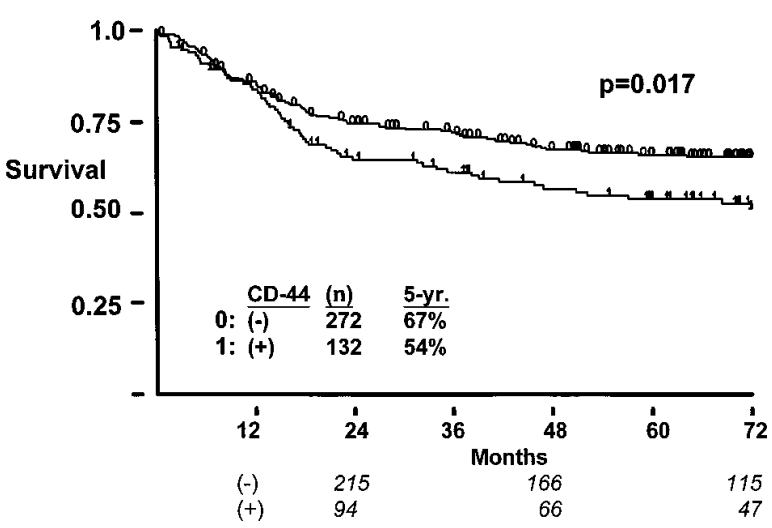

Fig 4. Kaplan-Meier analysis of cancer-specific survival for CD-44.

demonstrated that factor viii was superior to CD31 as an indicator of tumor angiogenesis and malignant potential (hazard ratio, 2.9). This study and numerous other studies have examined the relationship of a single oncogene or molecular marker on the survival in patients with lung cancer, without addressing the spectrum of oncogenic mechanisms in the molecular biology of lung cancer. ${ }^{14,19-21}$

In a previous study by 1 of the authors (D.H.H.), a panel of 4 molecular markers were applied to patients with stage I NSCLC: angiogenesis factor viii, p53, erbb2, and KI-67. ${ }^{13}$ Each factor had independent prognostic significance. Moreover, multivariable analysis demonstrated a powerful relationship among the number of molecular markers involved, in terms of survival. In patients with 0 markers, 1 marker, 2 markers, or 3 to 4 markers, the 5-year survival was $81 \%, 71 \%, 54 \%$, and $45 \%$, respectively. Finally, in a group of 244 


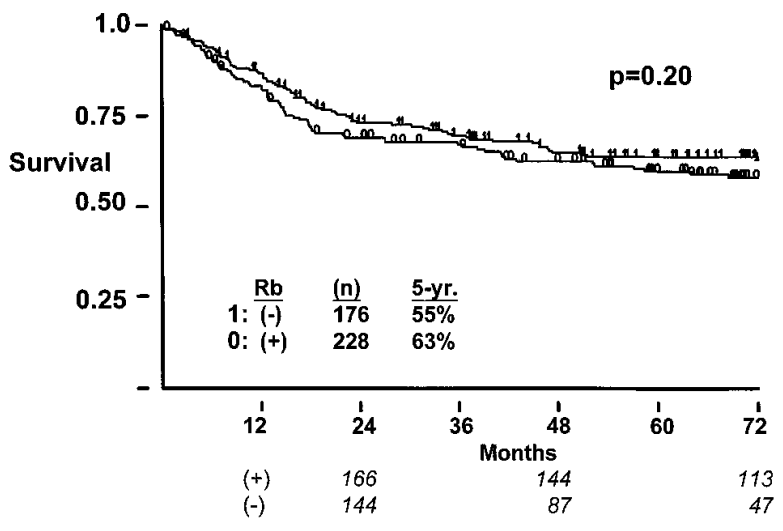

Fig 5. Kaplan-Meier analysis of cancer-specific survival for rb.

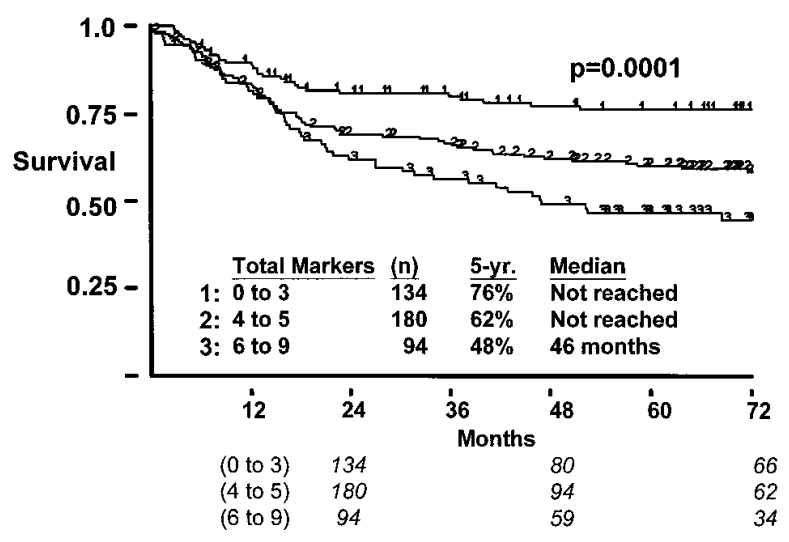

Fig 6. Analysis of cancer-specific survival according to the involvement of all molecular markers.

patients, a pathologic substaging system was developed, with 6 molecular markers. ${ }^{25}$ This staging system effectively stratifies patients in terms of risk of recurrence according to the number of markers involved. This study identified only 3 molecular markers that were statistically associated with recurrence: $\mathrm{p} 53, \mathrm{~K}-$ ras mutation, and the lack of $\mathrm{H}-$ ras $\mathrm{p} 21$ expression.

In the current study, we evaluate a panel of 10 molecular biologic markers in 408 patients with stage I NSCLC after complete resection, the largest population ever studied with molecular markers. To create a model of molecular biologic substaging, we have chosen markers that represent 5 separate oncogenic pathways: growth regulation, cell cycle regulation, apoptosis, angiogenesis, and metastatic adhesion.

EGFr, which is encoded by the protooncogene erb$\mathrm{b} 1$, is an important contributing factor in supporting

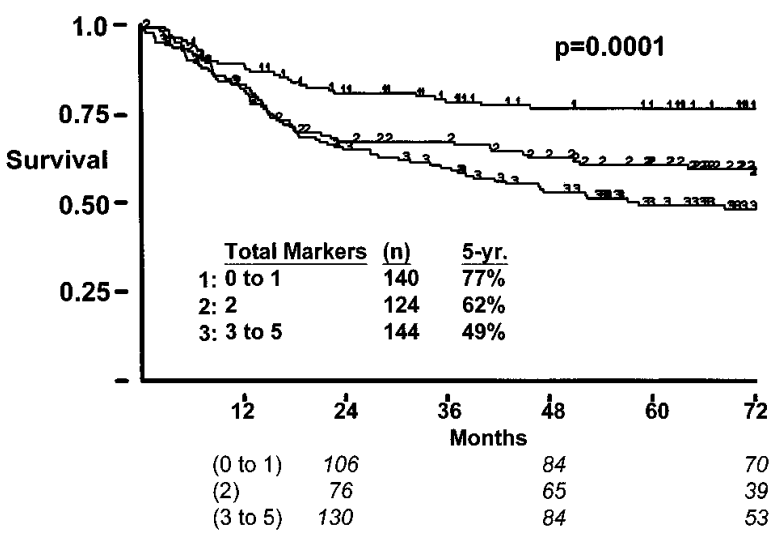

Fig 7. Analysis of cancer-specific survival according to the involvement of the 5 molecular markers that were significant in the multivariable analysis.

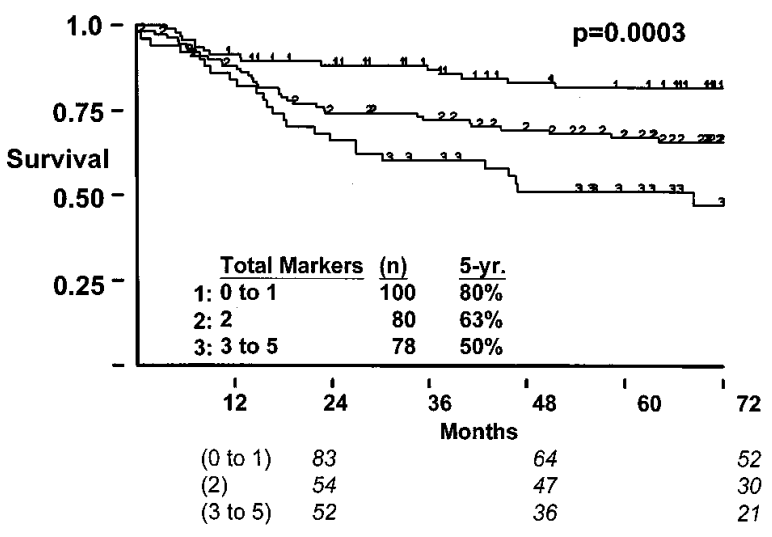

Fig 8. Analysis of cancer-specific survival among patients with $\mathrm{T} 1$ tumors according to the involvement of the 5 molecular markers that were significant in the multivariable analysis. Numbers on the Kaplan-Meier survival curves represent censors data. Data for the Kaplan-Meier survival estimates were truncated at 72 months survival for graph creation. There are 160 patients with more than 72 months survival (range, 72-201 months) who do not appear as tic marks on the curves.

uncontrolled tumor growth. The protooncogene $e r b-\mathrm{b} 2$, which shows extensive homology to erb-b1, encodes a membrane-associated tyrosine kinase that also serves as a growth factor receptor.

Cell cycle-regulating proteins have a direct effect on tumor clonal expansion. The rb interacts with p53 in regulating gene expression in the nucleus. The tumor proliferation marker KI-67 is a nonhistone nuclear protein that is expressed in cells that are near mitosis. The KI-67 proliferation index has been demonstrated to be associated with decreased survival in several stud- 
ies. ${ }^{13,15}$ Although the KI-67 proliferation index is related to the mitotic rate, KI-67 is a more prevalent proliferation marker and thus may be assessed in a greater number of patients.

Mutations in recessive oncogenes that code for apoptosis have been demonstrated to allow unregulated cell growth and contribute to the proliferation of malignant tumors. Normal p53 encodes a nuclear phosphoprotein required to regulate cell growth; mutated p53 stimulates unregulated cell growth and promotes malignancy. Similarly, bcl-2 is expressed in normal epithelial cells and encodes a protein that regulates cell viability and inhibits apoptosis. Although the overexpression of bcl-2 is associated with uncontrolled cell growth, it has been associated with improved survival when found in lung malignancies. ${ }^{26}$

Tumor cells initially obtain nutrition from central diffusion from surrounding pulmonary parenchyma. Once a colony outgrows its blood supply, central necrosis occurs. This ischemic process signals angiogenesis in the tumor, allowing capillary ingrowth into the tumor and further growth. The presence of angiogenesis factor viii has already been demonstrated as a powerful negative prognostic factor. Immunohistochemical analysis of factor viii allows for quantification of microvessel presence in a tumor. Studies have shown that the hottest area microvessel number is correlated with recurrence and death in patients with NSCLC. ${ }^{9,13} \mathrm{Al}$ though the microvessel count using factor viii and the histologic determination of pulmonary vascular invasion both have negative prognostic value, the prevalence of factor viii is significantly higher and allows application to a greater number of patients in a prognostic model.

The presence of blood vessels in the tumor allows cells to be shed into the circulation. The development of distant metastases requires the expression of cellular adhesion proteins, which are involved in local tumor invasion and metastases. CD-44 is an integral membrane glycoprotein that functions as a receptor for the extracellular matrix glycan, halyuronan. The loss of expression of blood group A antigen in patients with blood group A allows the expression of immature blood group antigens, which promotes metastatic adhesion and is postulated to confer metastatic potential. ${ }^{22}$ Aberrant glycosylation of mucins results in the expression of sialyl-Tn antigens in various malignant neoplasms ${ }^{23}$ and the expression of sialyl-Tn is associated with tumor progression, local invasion, and adhesion of tumor cells on the endothelium of distant vessels. ${ }^{24}$

In this molecular biologic risk model, 5 oncogenic mechanisms are represented by the panel of 10 markers
(Table I). Multivariable analysis confirmed prognostic value for 5 of the markers, including 1 marker from each oncogenic group (Table III).

The factor with the strongest independent prognostic value was p53 (hazard ratio, $1.63 ; P=.037$ ), supporting previous studies. Kaplan-Meier survival analysis demonstrates the negative prognostic value of the p53 overexpression, which is associated with 5-year survival of 52\%, compared with $70 \%$ in patients with normal p53 (Fig 1).

As in previous studies, hot area angiogenesis factor viii was associated with significantly decreased survival (hazard ratio, $1.47 ; P=.003$ ). The 5-year survival in patients with hot area angiogenesis was $56 \%$, compared with $70 \%$ in those patients with low factor viii counts (Fig 2).

The presence of the protooncogene $e r b$-b2 was also a strong negative prognostic factor (hazard ratio, 1.429; $P=.044)$. The 5-year survival of patients with $e r b-\mathrm{b} 2$ was $47 \%$, compared with $67 \%$ in patients without this marker (Fig 3).

The metastatic adhesion protein CD-44 was also negative prognostic factor (hazard ratio, 1.399; $P=.05$ ). The 5-year survival of patients with CD-44 was 54\%, compared with $67 \%$ in patients without CD-44 (Fig 4).

The presence of $\mathrm{rb}$, a cell cycle regulator, was demonstrated to be a positive prognostic factor in this study; the absence of rb was associated with significantly decreased survival; the 5-year survival of patients with rb was $63 \%$, compared with $55 \%$ in patients without rb (Fig 5).

In this study, the prevalence of the molecular markers ranged from $32 \%$ to $68 \%$, allowing most patients to be analyzed for 1 or more prognostic factors. When this group of patients was analyzed for the impact of the number of markers on survival, another important relationship appeared. Survival was strongly associated with the number of molecular risk factors, and 3 groups were created to stratify the risk of death. Patients with 0-3, 4-5, and 6-9 markers have 5-year survival of 76\%, $62 \%$, and $48 \%$, respectively (Fig 6). The Kaplan-Meier survival analysis was also performed for the panel of 5 markers that were found to be significant in the multivariable risk analysis. The 5-year survival for patients with 0-1 markers, 2 markers, and 3-5 markers was $77 \%, 62 \%$, and $58 \%$, respectively (Fig 7).

A panel of 10 molecular biologic markers were analyzed immunohistochemically in a large, single-institution population of patients with stage I NSCLC after complete resection. Immunohistochemical analysis is rapid, reproducible, relatively inexpensive, and generally available in most hospitals. This panel of markers 
represents 5 oncogenic mechanisms, and multivariable analysis identified 1 marker in each oncogenic group as a significant prognostic factor (p53, factor viii, erb-b2, $\mathrm{CD}-44$, and $\mathrm{rb}$ ). Although each factor alone carries independently significant prognostic information, risk stratification is further enhanced by analyzing patient survival according to the number of risk factors involved. In this model, patients with 3-5 molecular markers are at a significantly increased risk of cancerrelated death, despite complete surgical resection for stage I NSCLC. Although adjuvant therapy has not been demonstrated to be effective in patients with stage I disease after resection, it is postulated that risk stratification will identify a group of patients with a sufficiently elevated risk of death to justify adjuvant therapy.

This prognostic model uses molecular markers of 5 oncogenic mechanisms to analyze the risk of cancerrelated death. The advantages of this model include the ability to assess direct oncogenic mechanisms rather than the indirect indices of histopathologic variables and to analyze these variables in most patients in the population. Furthermore, the variables are related mechanistically, and the use of a group of factors to improve risk stratification is a logical approach. The prospective validation of this model is recommended before its application to assign adjuvant chemotherapy after complete resection in patients with completely resected NSCLC.

\section{REFERENCES}

1. Landis SH, Murray T, Bolden S, Wingo PA. Cancer statistics, 1998. CA Cancer J Clin 1998;48:6-29.

2. Mountain CF. Revisions in the International System for Staging Lung Cancer. Chest 1997;111:1710-7.

3. Harpole DH Jr, Herndon JE II, Young WG, Wolfe WG, Sabiston DC Jr. Stage I non-small cell lung cancer. Cancer 1995;76:787-96.

4. Mountain CF, Lukeman JM, Hammar SP. Lung cancer classification: the relationship of disease extent and cell type to survival in a clinical trials population. J Surg Oncol 1987;35:147-56.

5. Mountain CF. A new international staging system for lung cancer. Chest 1986;89:225S-33S.

6. Mountain CF. Value of the new TNM staging system for lung cancer. Chest 1989;96:47S-9S

7. Nesbitt JC, Putnam JB Jr, Walsh GL, Roth JA, Mountain CF. Survival in early-stage non-small cell lung cancer. Ann Thorac Surg 1995;60:466-72.

8. Ichinose Y, Hara N, Ohta M, Yano T, Maeda K, Asoh H, et al. Is $\mathrm{T}$ factor of the TNM staging system a predominant prognostic factor in pathologic stage I non-small cell lung cancer? J Thorac Cardiovasc Surg 1993;106:90-4.

9. Macchiarini P, Fontanini G, Hardin MJ, Chuanchieh H, Bigini D, Vignati S, et al. Blood vessel invasion by tumor cells predicts recurrence in completely resected T1 N0 M0 non-small cell lung cancer. J Thorac Cardiovasc Surg 1993;106:80-9.

10. Thomas PA, Rubinstein L. Cancer recurrence after resection: T1
N0 non-small cell lung cancer. Ann Thorac Surg 1990;49:2427.

11. Takise A, Kodama A, Shimosato Y, Watanabe S, Suemasu K. Histopathologic prognostic factors in adenocarcinoma of the lung periphery less than $2 \mathrm{~cm}$ in diameter. Cancer 1988;61:2083-8.

12. Pairolero PC, Williams DE, Bergstralh EJ, Piehler JM, Bernatz PE, Payne WS. Postsurgical stage I bronchogenic carcinoma: morbid implications of recurrent disease. Ann Thorac Surg 1984;38:331-6.

13. Harpole DH Jr, Richards WG, Herndon JE II, Sugarbaker DJ. Angiogenesis and molecular biologic substaging in patients with stage I non-small cell lung cancer. Ann Thorac Surg 1996;61:1470-6.

14. Harpole DH Jr, Marks JR, Richards WG, Herndon JE II, Sugarbaker DJ. Localized adenocarcinoma of the lung: oncogene expression of $e r b$-b2 and p53 in 150 patients. Clin Cancer Res 1995; 1:659-64.

15. Harpole DH Jr, Herndon JE II, Wolfe WG, Iglehart JD, Marks JR. A prognostic model of recurrence and death in stage I non-small cell lung cancer utilizing presentation, histopathology, and oncoprotein expression. Cancer Res 1995;55:51-6.

15. Strauss GM, Kwiatowski DJ, Harpole DH, Lynch TJ, Skarin AT, Sugarbaker DJ. Molecular and pathologic markers in stage I non-small cell carcinoma of the lung. J Clin Oncol 1995;13: 1265-79.

16. Matsuyama K, Chiba Y, Sasaki M, Tanaka H, Muraoka R, Tanigawa N. Tumor angiogenesis as a prognostic marker in operable non-small cell lung cancer. Ann Thorac Surg 1998;65:1405-9.

17. Bongiorno PF, Whyte RI, Lesser EJ, Moore JH, Orringer MB, Geer DG. Alterations of K-ras, p53, and erb-b2/neu in human lung adenocarcinomas. J Thorac Cardiovasc Surg 1994;107:590-5.

18. Duarte IG, Bufkin BL, Pennington MF, Gal AA, Cohen C, Kosinski AS, et al. Angiogenesis a predictor of survival after surgical resection for stage I non-small cell lung cancer. J Thorac Cardiovasc Surg 1998;115:652-9.

19. Kern JA, Schwartz DA, Nordberg JE. P185neu expression in human lung adenocarcinomas predicts shortened survival. Cancer Res 1990;50:5184-91.

20. Quinlan DC, Davidson AG, Summers CL, Warden HE, Doshi HM. Accumulation of $\mathrm{p} 53$ proteins correlates with a poor prognosis in human lung cancer. Cancer Res 1992;54:4828-31.

21. Rodenhuis S, Slebos RJC. Clinical significance of ras oncogene activation in human lung cancer. Cancer Res 1992;52:2665-9.

22. Matsumoto H, Muramatsu H, Shimotakahara T, Yanagi M, Nishijima H, Mitani N, et al. Correlation of ABH blood group carbohydrate antigens with metastatic potential in human lung carcinomas. Cancer 1993;72:75-81.

23. Terada T, Nakanuma Y. Expression of mucin carbohydrate antigens ( $\mathrm{T}, \mathrm{Tn}$, and sialyl Tn) and MUC-1 gene product in intraductal papillary mucinous neoplasms of the pancreas. Am J Clin Path 1996;105:613-20.

24. Soares R, Marinho A, Schmitt F. Expression of sialyl-Tn in breast cancer: correlation with prognostic parameters. Pathol Res Pract 1996; 192:1181-6.

25. Kwiatkowski DJ, Harpole DHH Jr, Godleski J, Herndon JE III, Shieh DB, Richards W, et al. Molecular pathologic substaging in 244 stage I non-small cell lung cancer patients: clinical implications. J Clin Oncol 1998; 16:2468-77.

26. Pezzella F, Turley H. Kuzu I, Tungekar MF, Dunnill MS, Pierce $\mathrm{CB}$, et al. Bcl-2 protein in non-small cell lung carcinoma. $\mathrm{N}$ Engl J Med 1993;329:690-4. 


\section{Discussion}

Dr John R. Benfield (Sacramento, Calif). I am delighted that the Program Committee chose to accept your paper because it is a preview to thoracic operations in the future. It is crucial that we not be intimidated by what you have presented today.

In a sense, your work is a follow-up to our 1988 presentation to The American Association for Thoracic Surgery (Benfield JR, Wain JC, Derrick M, et al. Biochemical and cytogenetic studies of human lung cancers. J Thorac Cardiovasc Surg 1988;96:840-8) and to Dr John Minna's presentation to this Association in 1990, which was titled "The Cellular and Molecular Biology of Lung Cancer." The fact that your multidisciplinary group is led by you and other surgeons will insure that thoracic surgeons continue to lead in the fight against lung cancer.

The hypothesis of your paper could be restated as follows: Each lung cancer has inherent biologic characteristics that allow one to predict its behavior and to plan therapy at the outset. In practicality, you are addressing the clinical frustration when we now operate on patients and believe them cured, only to find that at least $20 \%$ of such patients later experience recurrent cancer. What you are trying to find out is how to select patients for whom operation alone suffices as compared with those who require adjuvant or induction therapy even though their cancers appear to be early stage by current criteria. Is this a reasonable way to restate your hypothesis?

Dr D'Amico. Very elegant, yes.

Dr Benfield. I think we are looking forward, perhaps in another 5 years, to the opportunity to work up each patient's cancer in addition to studying the patient as we now do. Years hence, when a patient with lung cancer is referred to us, we will do a needle biopsy and from that specimen and from serologic specimens we will look at a molecular biologic panel of information. Such studies will tell us how aggressive each cancer is likely to be and what form of induction or adjuvant therapy should be given.

Your study is descriptive and not a study of mechanisms. Therefore, quite correctly, you have called for a prospective validation of the significance of the markers you have examined. There is a need to do this validation before you use your current results as the basis for a phase II adjuvant therapy study. In the meantime there is an opportunity to apply the techniques with which you have gained experience in samples obtained from patients enrolled in ongoing phase III lung cancer therapy studies.

To do the studies of mechanisms that I suggest, it will be difficult to get enough samples from patients alone. Thus I believe it would be helpful to carry forward in your work with animal models of lung cancer. Such models can be studied in serial fashion during carcinogenesis, and this should give more information than one could get from studies limited to patients with lung cancer.

How close are you to being able to apply your immunohistochemical techniques to fine-needle aspirate or cytologic specimens? What are your thoughts about the use of animal models of lung cancer as a supplement to the work that is ongoing at Duke?

Dr D'Amico. In terms of using the data that we have in retrospectively analyzing patients who have already been treated, our laboratory has concentrated on patients with stage I disease, so we're taking patients who have never been treated with adjuvant therapy. But, the preliminary work that our laboratory has done was used to construct the correlative science portion of cancer and leukemia group B study 9633, in which patients with T2 N0 are randomized to receive observation or adjuvant chemotherapy. All the patients, whether they are observed or treated, are going to be analyzed for the panel of molecular markers. So we will have these data, although the markers are not being used to assign chemotherapy; we will have in a prospective study the analysis of markers in patients who were observed or were given chemotherapy.

That addresses 1 of your points. To specifically address your questions, you outlined the model that we would have outlined in terms of how we would use this data to treat patients in the future, that is to know prospectively the molecular characteristics of the tumor from a needle biopsy and to use that to assign therapy before an operation. If for patients with stage I disease with high-risk markers it were to be shown that induction therapy were superior to adjuvant therapy as it has been shown for patients with stage IIIA disease, that would definitely be the way to go. There are some markers that we can analyze with small cytologic specimens; it is easy to analyze a small number of cells for P53 or for erb-b2, whereas analysis of other factors (such as angiogenesis) require that the tumor be intact to perform microvessel counts. So, some markers could be analyzed at the cytologic level; we would have to advance our techniques or use other ways such as another polymerase chain reaction to evaluate these markers. And that we can do.

To answer your second question in terms of actually getting at the mechanism of the molecular biology of lung cancer, I agree with you that that would require an animal model. The model that we have described is not really a mechanistic model; it is a descriptive model. We understand the difficulty in establishing animal models of lung cancer and that some of the difficulties that your work has shown can be overcome. And we understand the importance of animal models to get at the mechanistic level; we have not undertaken that, and I do not know if we will in the future. 